\title{
BLOQUEO DE LA VÍA AÉREA TRAS LA EXTUBACIÓN. REVISIÓN BIBLIOGRÁFICA
}

\section{Post-extubation airway obstruction. Literature review}

\author{
Álvaro SÁNCHEZ-TABERNERO ${ }^{1}$; José Luis PARDAL-REFOYO²; Jesús Javier CUELLO-AZCÁRATE ${ }^{1}$ \\ Complejo Asistencial de Zamora. ${ }^{1}$ Servicio de Anestesiología y Reanimación. ${ }^{2}$ Servicio de Otorrinolaringología. Zamora. \\ España. \\ Correspondencia: alvarosanchezt@hotmail.com
}

Fecha de recepción: 2 de octubre de 2016

Fecha de aceptación: 19 de octubre de 2016

Fecha de publicación: 29 de octubre de 2016

Fecha de publicación del fascículo: 1 de marzo de 2017

Conflicto de intereses: Los autores declaran no tener conflictos de intereses

Imágenes: Los autores declaran haber obtenido las imágenes con el permiso de los pacientes

Política de derechos y autoarchivo: se permite el autoarchivo de la versión post-print (SHERPA/RoMEO)

Licencia CC BY-NC-ND. Licencia Creative Commons Atribución-NoComercial-SinDerivar 4.0 Internacional

๔ Universidad de Salamanca. Su comercialización está sujeta al permiso del editor

RESUMEN

\begin{abstract}
Introducción y objetivo: El bloqueo de la vía aérea (BVA) tras la extubación en cualquier cirugía es un evento crítico con baja incidencia, potencialmente grave, que puede precisar reintubación o traqueotomía, en el que frecuentemente el otorrinolaringólogo es requerido. Objetivo: Conocer la prevalencia de BVA y sus causas mediante revisión bibliográfica sistemática. Método: Revisión bibliográfica en PubMed, Cochrane y Scopus de ensayos clínicos, metanálisis, revisiones y series de casos y control sobre obstrucción de la vía aérea tras extubación que precisa reintubación en adultos. Resultados: Se seleccionaron 6 estudios y una guía de práctica clínica. La causa más frecuente de fallo de extubación es el bloqueo de la vía aérea por diversas causas (debilidad muscular faríngea - frecuentemente por efecto residual farmacológico-, laringoespasmo, parálisis de cuerdas vocales, edema de vías respiratorias superiores, hematoma cervical postoperatorio, cuerpos extraños o secreciones). La mayoría de los casos de reintubación ocurrieron en las 2 horas tras la extubación. Conclusiones: La causa más frecuente de fallo tras la extubación en anestesia general es el bloqueo de la vía aérea generalmente provocado por efecto de bloqueo neuromuscular residual. El riesgo de BVA se incrementa en la cirugía de la vía aérea y de cabeza y cuello. Las guías de intubación difícil han mejorado la actuación y reducido los eventos adversos y es necesario implementar estrategias similares en la extubación. El procedimiento de extubación y reintubación debe estar documentado. Los grupos de trabajo sobre la vía aérea han de ser multidisciplinares e incluir especialistas en otorrinolaringología.
\end{abstract}

PALABRAS CLAVE vía aérea; obstrucción de la vía aérea; extubación; intubación; fallo en la extubación

SUMMARY Introduction and objective: airway obstruction after extubation in any surgery is a critical event with low incidence, which may require reintubation or tracheostomy, which often 
otolaryngologist is required. Objective: To determine the prevalence of BVA and its causes through systematic literature review. Method: Literature review in PubMed, Scopus and Cochrane clinical trials, meta-analysis, reviews and case series and control over airway obstruction after extubation that requires reintubation in adults. Results: 6 studies and one clinical practice guidelines were selected. The most common cause of extubation failure is blocking the airway for various reasons (pharyngeal muscle weakness residual effect -often farmacologycal-, laryngospasm, vocal cord paralysis, edema of upper respiratory tract, cervical postoperative hematoma, foreign bodies or secretions). Most cases of re-intubation occurred within 2 hours after extubation. Conclusions: The most common cause of failure after general anesthesia extubation is blocking the airway generally caused by residual neuromuscular blocking effect. Airway obstruction risk increases in airway and head and neck surgery. Difficult intubation guidlines have improved performance and reduced adverse events and similar strategies must be implemented in extubation. The procedure extubation and reintubation should be documented. Working groups airway must be multidisciplinary and include specialists in otolaryngology.

KEYWORDS

airway; extubation airway; obstruction airway; extubation; intubation; extubation failure

\section{INTRODUCCIÓN}

Las complicaciones respiratorias son el segundo problemas más frecuente, tras las náuseas y vómitos y el bloqueo de la vía aérea (BVA) tras la extubación en cualquier tipo de cirugía es un evento crítico, potencialmente muy grave, que puede provocar la muerte o secuelas graves al paciente [1,2]. Se define extubación (término MeSH introducido en 2012, airway extubation) como la extracción del tubo endotraqueal del paciente y se define fallo de extubación a la imposibilidad de tolerar la ausencia de tubo endotraqueal habitualmente tratado con la reintubación [3].

La extubación traqueal es un paso crítico tras la que se debe mantener la vía aérea permeable y protegida de aspiraciones, y la ventilación (oxigenación pulmonar) y, en pacientes de alto riesgo —obesidad, síndrome de apnea obstructiva del sueño o dificultad durante la intubación- los incidentes de BVA en la extubación son causa frecuente de reclamación por muerte o daño cerebral [4].

La causa más común de obstrucción de la vía respiratoria superior en la unidad de reanimación posanestésica (URPA) es la debilidad muscular faríngea, generalmente debida a bloqueo neuromuscular residual, seguido de efectos residuales de los otros anestésicos y de los opioides [5-7].

Otras causas de BVA tras la extubación son el laringoespasmo, la parálisis laríngea bilateral (por lesión bilateral del nervio laríngeo recurrente generalmente secundario a tiroidectomía), el edema de vía aérea superior (por posición en Trendelenburg, exceso de fluidoterapia, anafilaxia), traumatismos sobre la vía aérea (en la intubación, debido a cirugía en la vía aérea), hematoma sofocante o cuerpos extraños en la vía aérea [8-17].

La incidencia global de BVA tras la extubación es baja, en torno al $0,17 \%$ [18] por lo que el mejor método de investigación es la revisión bibliográfica sistemática.

El objetivo de este trabajo es conocer la prevalencia del bloqueo de la vía aérea sucedido tras la extubación por anestesia general tras una intervención quirúrgica mediante revisión bibliográfica sistemática.

\section{MATERIAL Y MÉTODO}

Se realizó una búsqueda bibliográfica sistemática en las bases de datos PubMed, Cochrane Library y Scopus con las palabras clave o descriptores y estrategias de búsqueda que se resumen en el diagrama de la figura 1. Los criterios de inclusión fueron: artículos en lengua española o inglesa, con resumen disponible, sobre bloqueo de la vía aérea en humanos adultos y reintubación. Se incluyeron ensayos clínicos, series de casos y control, artículos de revisión, metanálisis y guías de práctica clínica.

\section{RESULTADOS}

Para el estudio cualitativo se seleccionaron 7 trabajos que cumplían los criterios de inclusión [19-25], tal como se resume en la figura 1 - solo se incluyeron los estudios que específicamente abordaban el tema del fallo de la extubación tras anestesia que precisó reintubación, no cumplieron los criterios ni los metanálisis ni los ensayos clínicos revisados-

En la tabla 1 se resumen los resultados más relevantes de la revisión. 


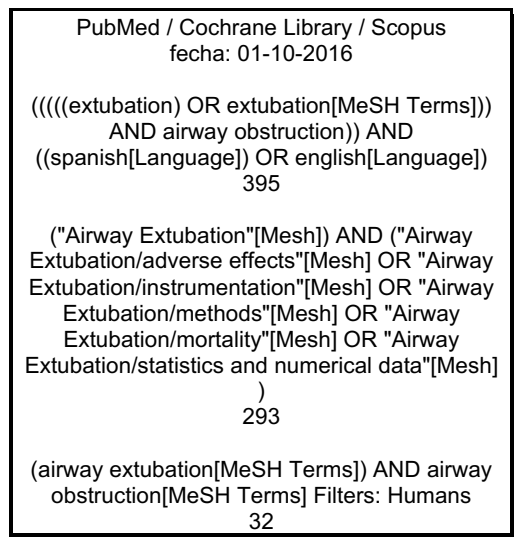

(((()((EXTUBATION) OR EXTUBATION[MeSH Terms])) AND AIRWAY OBSTRUCTION)) AND ((SPANISH[Language]) OR

ENGLISH[Language]))) AND adult[MeSH Terms]
159

\begin{tabular}{|c|}
\hline Selección inicial \\
41 \\
\\
Meta-analysis (5) \\
Clinical Trial (20) \\
Review (16) \\
Guidelines (1) \\
\hline
\end{tabular}

Estudios seleccionados para estudio cualitativo (Review)

Mathew et al., 1990 [19]

Chinachoti et al., 2005 [20]

Chinachoti et al., 2008 [21]

Ting et al., 2010 [22]

Cook et al., 2011 [23]

Huan-Tang et al., 2013 [24]

Popat et al., 2012 [25]

Figura 1. Obstrucción de la vía aérea tras la extubación. Diagrama con la búsqueda bibliográfica y selección de artículos.

Los incidentes más frecuentes tras la extubación son la hipoventilación por alteración de los reflejos en la vía aérea (por exceso -laringoespasmo, broncoespasmo, tos- o por defecto -efecto de opioides, efecto farmacológico residual, movimiento paradójico de las cuerdas vocales-) y el BVA -secreciones, sangre, cuerpo extraño, edema, hematoma extrínseco compresivo, parálisis bilateral de cuerdas vocales-.

La mayoría de las reintubaciones por fallos de extubación se producen en el quirófano
$[20,22,24]$ en las 2 primeras horas tras la extubación [19,21].

Los factores de riesgo que se relacionan con los fallos en la extubación son la alteración consciencia, EPOC, neumonía, SRIS (síndrome de respuesta inflamatoria sistémica) - fiebre mayor de $38^{\circ} \mathrm{C}$ o hipotermia, taquipnea o pCO2 menor de $32 \mathrm{mmHg}$, taquicardia, leucocitosis o leucopenia o desviación izquierda-, saturación O2 menor del 95\%, hipotermia, algunos relajantes musculares como el rocuronio, cirugía de vía aérea y cirugía de cabeza y cuello y fallos humanos -inexperiencia- $[21,24]$.

Tres aspectos relacionados con la vía aérea incrementan el riesgo de fallo en la reintubación tras la extubación: la vía aérea difícil tras la primera intubación —más frecuente en obesidad, SAOS o pacientes con alto riesgo de aspiración-, el deterioro perioperatorio de la vía aérea - por distorsión de la anatomía, edema, hemorragia, lesión laríngea-y la dificultad para el acceso a la vía aérea tras la cirugía —por cambios quirúrgicos, colgajos o fijación cervical- [24]. Las consecuencias de los incidentes relacionados con los fallos en la extubación y reintubación son el incremento de la estancia hospitalaria, el incremento de la estancia en $\mathrm{UCl}$, la necesidad de realizar traqueotomía y el incremento de la mortalidad [24].

\section{DISCUSIÓN}

Las referencias al procedimiento de extubación y reintubación en caso de fallo en la extubación son indirectas, generalmente incluidas en guías sobre vía aérea difícil pero sin establecer un procedimiento sistematizado hasta la publicación de la difficult airway society guidelines for the management of tracheal extubation [25] (Tabla 2).

En la tabla 3 se resumen los aspectos más importantes para la discusión tras los resultados obtenidos en la revisión bibliográfica.

EI BVA tras la extubación en cualquier tipo de cirugía es un evento poco frecuente, crítico y potencialmente muy grave que puede ocasionar daño pulmonar, cerebral o la muerte. Influyen factores fisiopatológicos, farmacológicos y humanos [25]. En la mayoría de los casos el BVA se produce en las primeras 2 a 6 horas tras la extubación y para resolverlo se necesitará reintubación o traqueotomía. 
Tabla 1. Obstrucción de la vía aérea tras la extubación. Reintubación tras la extubación en anestesia general. Resumen de los artículos seleccionados.

\begin{tabular}{|c|c|c|c|}
\hline Autor & Método & $\begin{array}{l}\text { Incidente que precisa } \\
\text { reintubación }\end{array}$ & Observaciones \\
\hline $\begin{array}{l}\text { Mathew et al., } \\
1990[19]\end{array}$ & retrospectivo & $\begin{array}{l}26 / 13593=0,19 \% \\
20 / 26=76,9 \% \text { en primera } \\
\text { hora } \\
54 \% \text { edad }<3 \text { y }>60 \text { años } \\
23 \% \text { cirugía ORL }\end{array}$ & $\begin{array}{l}\text { Causas más frecuentes: excesiva sedación / efecto residual } \\
\text { farmacológico / persistencia de miorrelajación (18/26=69\% } \\
\text { causa anestésica). } \\
\text { Fluidoterapia inadecuada. } \\
\text { Obstrucción de la vía aérea superior. } \\
\text { Ningún paciente tuvo secuelas }\end{array}$ \\
\hline $\begin{array}{l}\text { Chinachoti et al., } \\
2005[20]\end{array}$ & retrospectivo & $\begin{array}{l}234 / 86667=0,27 \% \\
158 / 234=67,5 \% \text { en quirófano } \\
\text { o URPA } \\
\text { En }<6 \mathrm{~h} \\
83 / 234=35,5 \% \text { a los } 10 \\
\text { minutos de extubación } \\
\text { Edad de mayor riesgo entre } \\
<1 \text { y }>70 \text { años }\end{array}$ & $\begin{array}{l}\text { Bloqueo neuromuscular (53\%) } \\
\text { Agente anestésico residual (57\%) (53-57\%), } \\
\text { Bloqueo de vía aérea ( } 31 \%) \\
\text { Inestabilidad hemodinámica (26.3\%). }\end{array}$ \\
\hline $\begin{array}{l}\text { Chinachoti et al., } \\
2008 \text { [21] }\end{array}$ & retrospectivo & $\begin{array}{l}184 \text { casos } \\
90,2 \% \text { en }<2 \mathrm{~h} \\
\text { Hipoventilación }(58,1 \%) \\
\text { Bloqueo de la vía aérea } \\
(39,6 \%)\end{array}$ & $\begin{array}{l}99,2 \% \text { incidentes anestésicos } \\
54,5 \% \text { incidentes no anestésicos } \\
99,2 \% \text { inadecuado manejo } \\
\text { El incidente es prevenible: } \\
\text { en el } 99,2 \% \text { de los incidentes relacionados con la anestesia } \\
\text { y en el } 54,5 \% \text { de los incidentes no relacionados directamente } \\
\text { con la anestesia } \\
99,2 \% \text { se relacionan con falta de experiencia o decisión } \\
\text { incorrecta }\end{array}$ \\
\hline $\begin{array}{l}\text { Ting et al., } 2010 \\
\text { [22] }\end{array}$ & retrospectivo & $\begin{array}{l}83 / 137.866=0,06 \% \\
65(78,3 \%) \text { en quirófano } \\
18(21,7 \%) \text { en URPA }\end{array}$ & $\begin{array}{l}\text { Grupos de riesgo: } \\
\text { EPOC preoperatoria (OR: 7,17; IC 95\%, 1,98-26,00). } \\
\text { Neumonía (OR: 7,94 IC 95\%: 1,93-32,78). } \\
\text { Ascitis (OR: 13,76; IC 95\%: 1,08-174,74). } \\
\text { Síndrome de respuesta inflamatoria sistémica (SRIS) (OR: } \\
\text { 11.90, IC 95\%: 2,63-53,86). } \\
\text { Cirugía de cabeza y cuello (OR: 3,43; IC 95\%: 1.24-9.50). } \\
\text { Cirugía en la vía aérea (OR: 5.98; IC 95\%: 1.59-22.60). }\end{array}$ \\
\hline $\begin{array}{l}\text { Cook et al., } 2011 \\
\text { [23] }\end{array}$ & retrospectivo & $\begin{array}{l}184 / 2.872 .600=0,0064 \% \\
46 \text { incidentes por cada millón } \\
\text { de anestesiados ( } 1 \text { por cada } \\
22.000 \text { ) } \\
133 \text { eventos relacionados } \\
\text { con BVA tras anestesia } \\
\text { general }\end{array}$ & $\begin{array}{l}16 \text { muertes } \\
13 \text { daño cerebral } \\
\text { Tasa de mortalidad: } 5,6 \text { por millón de procesos } \\
\text { Se estima que se comunica sólo el } 25 \% \text { de los incidentes } \\
\text { relevantes }\end{array}$ \\
\hline $\begin{array}{l}\text { Huan-Tang et al., } \\
2013 \text { [24] }\end{array}$ & $\begin{array}{l}\text { Casos } \\
\text { controles }\end{array}$ & $\begin{array}{l}130 / 227876=0,057 \% \\
102(78,5 \%) \text { en quirófano } \\
28 \text { en URPA } \\
\text { Factores de riesgo: } \\
\text { Alteración consciencia } \\
\text { EPOC } \\
\text { Neumonía } \\
\text { SRIS } \\
\text { Saturación O2<95\% } \\
\text { Hipotermia } \\
\text { Cirugía de vía aérea } \\
\text { Cirugía de cabeza y cuello }\end{array}$ & $\begin{array}{l}\text { Causas: } \\
\text { EPOC (OR: 4.30), Neumonía (OR: 6.60), Ascitis (OR: 4.86), } \\
\text { SRIS (OR: 7.52), } \\
\left.\text { Hipotermia (<35 }{ }^{\circ} \text {; OR: } 2.45\right), \\
\text { Rocuronium como relajante (OR: 1.90), } \\
\text { Inexperiencia (OR: } 3.44) \text {, } \\
\text { Cirugía en la vía aérea (OR: 4.34) } \\
\text { Consecuencias: } \\
\text { Incremento de estancia hospitalaria / estancia en UCl (OR: } \\
\text { 2.46) } \\
\text { Traqueotomía y mortalidad (OR: } 58.52)\end{array}$ \\
\hline
\end{tabular}

BVA: bloqueo de la vía aérea; OR: odds ratio; SRIS: síndrome de respuesta inflamatoria sistémica 
El procedimiento de extubación debe estar documentado así como los protocolos que deben seguirse en caso de fallo potencial de la extubación (reintubación, extubación diferida, traqueotomía electiva) [25].

La traqueotomía electiva debe plantearse en caso probabilidad de deterioro de la vía aérea en el postoperatorio (edema) evaluando el grado de compromiso de la vía aérea al final de la cirugía, la capacidad de acceso a la vía aérea en caso de bloqueo tras la extubación, la duración esperada de compromiso de la vía aérea y la probabilidad de que se produzca una extubación no planificada y no poder reintubar [25]

Hay dos aspectos importantes deducidos de esta revisión sobre los que debemos reflexionar. Por una parte, que en los grupos de trabajo no se incluyen especialistas en otorrinolaringología y, por otra, la escasa referencia a la traqueotomía como técnica en los casos de fallo de la reintubación.

Respecto a los aspectos metodológicos, faltan trabajos con suficiente evidencia que orienten a las mejores prácticas en la extubación.

Tabla 2. Bloqueo de la vía aérea tras la extubación. Guías con referencia a los fallos de extubación [25].

\begin{tabular}{|l|l|}
\hline 1998 & $\begin{array}{l}\text { Canadian Airway Focus Group's } \\
\text { recommendations for the management of } \\
\text { the unanticipated difficult airway }\end{array}$ \\
\hline 2003 & $\begin{array}{l}\text { American Society of Anesthesiologists } \\
\text { (ASA) difficult airway guidelines }\end{array}$ \\
\hline 2005 & $\begin{array}{l}\text { Societa Italiana Anaesthesia Analgesia } \\
\text { Rianimazione Terapia Intensiva (SIAARTI) } \\
\text { Recommendations for airway control and } \\
\text { difficult airway management }\end{array}$ \\
\hline 2004 & $\begin{array}{l}\text { Difficult Airway Society (DAS) difficult } \\
\text { intubation guidelines of mention the need for } \\
\text { a pre-formulated extubation plan } \\
\text { Difficult Airway Society Extubation } \\
\text { Guidelines Group. Difficult airway society } \\
\text { guidelines for the management of tracheal } \\
\text { extubation }\end{array}$ \\
\hline
\end{tabular}

\section{CONCLUSIONES}

La causa más frecuente de fallo tras la extubación en anestesia general es el bloqueo de la vía aérea generalmente provocado por efecto de bloqueo neuromuscular residual. El riesgo de BVA se incrementa en la cirugía de la vía aérea y de cabeza y cuello.
Las guías de intubación difícil han mejorado la actuación y reducido los eventos adversos y es necesario implementar estrategias similares en la extubación.

El procedimiento de extubación y reintubación debe estar documentado.

Los grupos de trabajo sobre la vía aérea han de ser multidisciplinares e incluir especialistas en otorrinolaringología.

Tabla 3. Bloqueo de la vía aérea tras la extubación. Resumen [19-25].

\begin{tabular}{|c|c|}
\hline $\begin{array}{l}\text { BVA tras } \\
\text { extubación tras } \\
\text { anestesia } \\
\text { general }\end{array}$ & Incidencia: 0,6 al 1,9 \%o \\
\hline Lugar & Quirófano 78\% / URPA 20\% \\
\hline Tiempo & $<6$ horas \\
\hline Edad & $<1$ años $->70$ años \\
\hline Grupos de riesgo & $\begin{array}{l}\text { Alteración consciencia / } \\
\text { Hipotermia } \\
\text { EPOC / SAOS / Neumonía / } \\
\text { Obesidad } \\
\text { SRIS } \\
\text { Saturación O2<95\% } \\
\text { Cirugía de vía aérea / cabeza y } \\
\text { cuello }\end{array}$ \\
\hline Causas & $\begin{array}{l}\text { Bloqueo neuromuscular }(53 \%) \\
\text { Agente anestésico residual } \\
(57 \%) \\
\text { Bloqueo de vía aérea ( } 31 \%) \\
\text { Inestabilidad hemodinámica } \\
(26.3 \%) \\
\text { Laringoespasmo } 8,7 \% \text { o }\end{array}$ \\
\hline Consecuencias & $\begin{array}{l}\text { Fallo en la reintubación / } \\
\text { traqueotomía / estancia } \\
\text { hospitalaria o en UCI } \\
\text { Edema pulmonar secundario } \\
\text { Muerte cerebral }\end{array}$ \\
\hline $\begin{array}{l}\text { Fallos de } \\
\text { reintubación }\end{array}$ & Incidencia desconocida \\
\hline $\begin{array}{l}\text { Participación de } \\
\text { especialistas en } \\
\text { ORL }\end{array}$ & No \\
\hline Evitabilidad & $>90 \%$ evitables [21] \\
\hline
\end{tabular}

EPOC: enfermedad pulmonar obstructiva crónica; SAOS: síndrome de apnea obstructiva del sueño; SRIS: síndrome de respuesta inflamatoria sistémica; URPA: unidad de reanimación posanestésica 
BIBLIOGRAFÍA

1. Hines R, Barash PG, Watrous G, O'Connor T. Complications occurring in the postanesthesia care unit: a survey. Anesth Analg 1992 Apr;74(4):503-9.

2. Kluger MT, Bullock MFM. Recovery room incidents: a review of 419 reports from the Anaesthetic Incident Monitoring Study (AIMS). Anaesthesia 2002;57(11):1060-6.

3. Epstein SK. Decision to extubate. Intensive Care Med 2002 May;28(5):535-46.

4. Peterson GN, Domino KB, Caplan RA, Posner KL, Lee LA, Cheney FW. Management of the difficult airway: a closed claims analysis. Anesthesiology 2005;103(1):33-9.

5. Murphy GS, Brull SJ. Residual neuromuscular block: lessons unlearned. Part I: definitions, incidence, and adverse physiologic effects of residual neuromuscular block. Anesth Analg 2010;111(1):120-8.

6. Brull SJ, Murphy GS. Residual neuromuscular block: lessons unlearned. Part II: methods to reduce the risk of residual weakness. Anesth Analg 2010;111(1):12940.

7. Grosse-Sundrup M, Henneman JP, Sandberg WS, Bateman BT, Uribe JV, Nguyen NT, et al. Intermediate acting nondepolarizing neuromuscular blocking agents and risk of postoperative respiratory complications: prospective propensity score matched cohort study. BMJ 2012;345:e6329.

8. Boyd M, Chatterjee A, Chiles C, Chin R. Tracheobronchial foreign body aspiration in adults. South Med J 2009;102(2):171-4.

9. Swanson KL, Edell ES. Tracheobronchial foreign bodies. Chest Surg Clin N Am 2001;11(4):861-72.

10. Sagi HC, Beutler W, Carroll E, Connolly PJ. Airway complications associated with surgery on the anterior cervical spine. Spine (Phila Pa 1976) 2002 1;27(9):949-53.

11. Wittekamp BHJ, van Mook WNKA, Tjan DHT, Zwaveling JH, Bergmans DCJJ. Clinical review: post-extubation laryngeal edema and extubation failure in critically ill adult patients. Crit Care 2009;13(6):233.
12. Sanapala A, Nagaraju M, Rao LN, Nalluri K. Management of bilateral recurrent laryngeal nerve paresis after thyroidectomy. Anesth essays Res;9(2):251-3.

13. Rubin $A D$, Sataloff RT. Vocal fold paresis and paralysis. Otolaryngol Clin North Am 2007;40(5):1109-31, viii-ix.

14. Weber $\mathrm{S}$. Traumatic complications of airway management. Anesthesiol Clin North America 2002;20(3):503-12.

15. Sue RD, Susanto I. Long-term complications of artificial airways. Clin Chest Med 2003;24(3):457-71.

16. Rosenthal LHS, Benninger MS, Deeb RH. Vocal fold immobility: a longitudinal analysis of etiology over 20 years. Laryngoscope 2007;117(10):1864-70.

17. Visvanathan T, Kluger MT, Webb RK, Westhorpe RN. Crisis management during anaesthesia: laryngospasm. Qual Saf Health Care 2005;14(3):e3.

18. Lee PJ, MacLennan A, Naughton NN, O'Reilly M. An analysis of reintubations from a quality assurance database of 152,000 cases. J Clin Anesth 2003;15(8):575-81.

19. Mathew JP, Rosenbaum SH, O'Connor T, Barash PG. Emergency tracheal intubation in the postanesthesia care unit: physician error or patient disease? Anesth Analg 1990;71(6):691-7.

20. Chinachoti $T$, Chau-in W, Suraseranivongse S, Kitsampanwong W, Kongrit P. Postoperative reintubation after planned extubation in Thai Anesthesia Incidents Study (THAl Study). J Med Assoc Thai. 2005;88 Suppl 7:S84--94.

21. Chinachoti T, Poopipatpab S, Buranatrevedhya S, Taratarnkoolwatana K, Werawataganon T, Jantorn P. The Thai Anesthesia Incident Monitoring Study (Thai AIMS) of post anesthetic reintubation: an analysis of 184 incident reports. J Med Assoc Thai. 2008;91(11):1706-13.

22. Ting PC, Chou AH, Yang MW, Ho AC-Y, Chang CJ, Chang SC. Postoperative reintubation after planned extubation: A review of 137,866 general anesthetics from 2005 to 2007 in a Medical Center of Taiwan. Acta Anaesthesiol Taiwanica 2010;48(4):167-71. 
23. Cook TM, Woodall N, Frerk C, Fourth National Audit Project. Major complications of airway management in the UK: results of the Fourth National Audit Project of the Royal College of Anaesthetists and the Difficult Airway Society. Part 1: anaesthesia. Br J Anaesth 2011;106(5):617-31.

24. Lin $\mathrm{H}-\mathrm{T}$, Ting $\mathrm{P}-\mathrm{C}$, Chang $\mathrm{W}-\mathrm{Y}$, Yang $\mathrm{M}$, Chang C-J, Chou A-H. Predictive risk index and prognosis of postoperative reintubation after planned extubation during general anesthesia: A single-center retrospective case-controlled study in Taiwan from 2005 to 2009. Acta Anaesthesiol Taiwanica 2013;51(1):3-9.

25. Difficult Airway Society Extubation Guidelines Group, Popat M, Mitchell V, Dravid R, Patel A, Swampillai C, et al. Difficult Airway Society Guidelines for the management of tracheal extubation. Anaesthesia 2012;67(3):318-40. 